Review Article

\title{
Three-Dimensional Organoid System Transplantation Technologies in Future Treatment of Central Nervous System Diseases
}

\author{
NaiLi Wei, ${ }^{1,2}$ ZiFang Quan, ${ }^{3}$ Hailiang Tang, ${ }^{1}$ and JianHong Zhu ${ }^{1}$ \\ ${ }^{1}$ Department of Neurosurgery, Fudan University Huashan Hospital, National Key Laboratory of Medical Neurobiology, \\ The Institutes of Brain Science and The Collaborative Innovation Center for Brain Science, Shanghai Medical College, \\ Fudan University, Shanghai 200040, China \\ ${ }^{2}$ Department of Neurosurgery, The Second Hospital of Lanzhou University, Lanzhou, Gansu 730030, China \\ ${ }^{3}$ Department of Neurology, The First Affiliated Hospital, University of South China, Hengyang, Hunan 421001, China
}

Correspondence should be addressed to Hailiang Tang; tangtang052105192@gmail.com and JianHong Zhu; jzhu@fudan.edu.cn Received 24 February 2017; Revised 24 May 2017; Accepted 8 June 2017; Published 20 August 2017

Academic Editor: Heinrich Sauer

Copyright (C) 2017 NaiLi Wei et al. This is an open access article distributed under the Creative Commons Attribution License, which permits unrestricted use, distribution, and reproduction in any medium, provided the original work is properly cited.

In recent years, scientists have made great achievements in understanding the development of human brain and elucidating critical elements of stepwise spatiotemporal control strategies in neural stem cell specification lineage, which facilitates successful induction of neural organoid in vitro including the cerebral cortex, cerebellar, neural tube, hippocampus cortex, pituitary, and optic cup. Besides, emerging researches on neural organogenesis promote the application of $3 \mathrm{D}$ organoid system transplantation in treating central nervous system (CNS) diseases. Present review will categorize current researches on organogenesis into three approaches: (a) stepwise, direct organization of region-specific or population-enriched neural organoid; (b) assemble and direct distinct organ-specific progenitor cells or stem cells to form specific morphogenesis organoid; and (c) assemble embryoid bodies for induction of multilayer organoid. However, the majority of these researches focus on elucidating cellular and molecular mechanisms involving in brain organogenesis or disease development and only a few of them conducted for treating diseases. In this work, we will compare three approaches and also analyze their possible indications for diseases in future treatment on the basis of their distinct characteristics.

\section{Introduction}

Stem cell therapy provides with an alternative and the last resort for curing many diseases in an extensive CNS spectrum of disease. However, poor clinical efficiency casts a showdown for stem cell therapy. In general, if neural stem cells take action, they should undergo three steps: proliferate and differentiate into due neural cells, migrate and distribute to accurate location, and integrate into host tissue and form synapse connection [1]. Unfortunately, this process usually takes several weeks [2]. Only a fringe of them finally survives and takes action. Currently, researchers and scientists devote themselves to improving the efficiency through optimizing various parameters such as engineering ideal matrices, suitable delivery approaches, and improving differentiation efficacy. However, stem cells are poorly manipulated in vivo. Once they are engrafted in vivo, they lose our control. Therefore, high-survival rate and stable environment in vivo are critical for stem cell transplantation. In order to solve these problems, organoid-like tissue might provide us with a promising approach.

Organoid is defined as a multicellular formation that spontaneously develops and self-organizes from stem cells or organ progenitors, resembling the structure and function of an organ in vivo [3]. Organoid system recapitulates the process of organogenesis in vivo and harbors stable hemostasis and architecture. Different from traditional stem cell therapy which always concentrated on specific populations of stem cells or progenitor cells, organoids provide with a complete set of cell types of an organ [3-6]. This novel 
Lineage specification of brain organoid and neural subtype
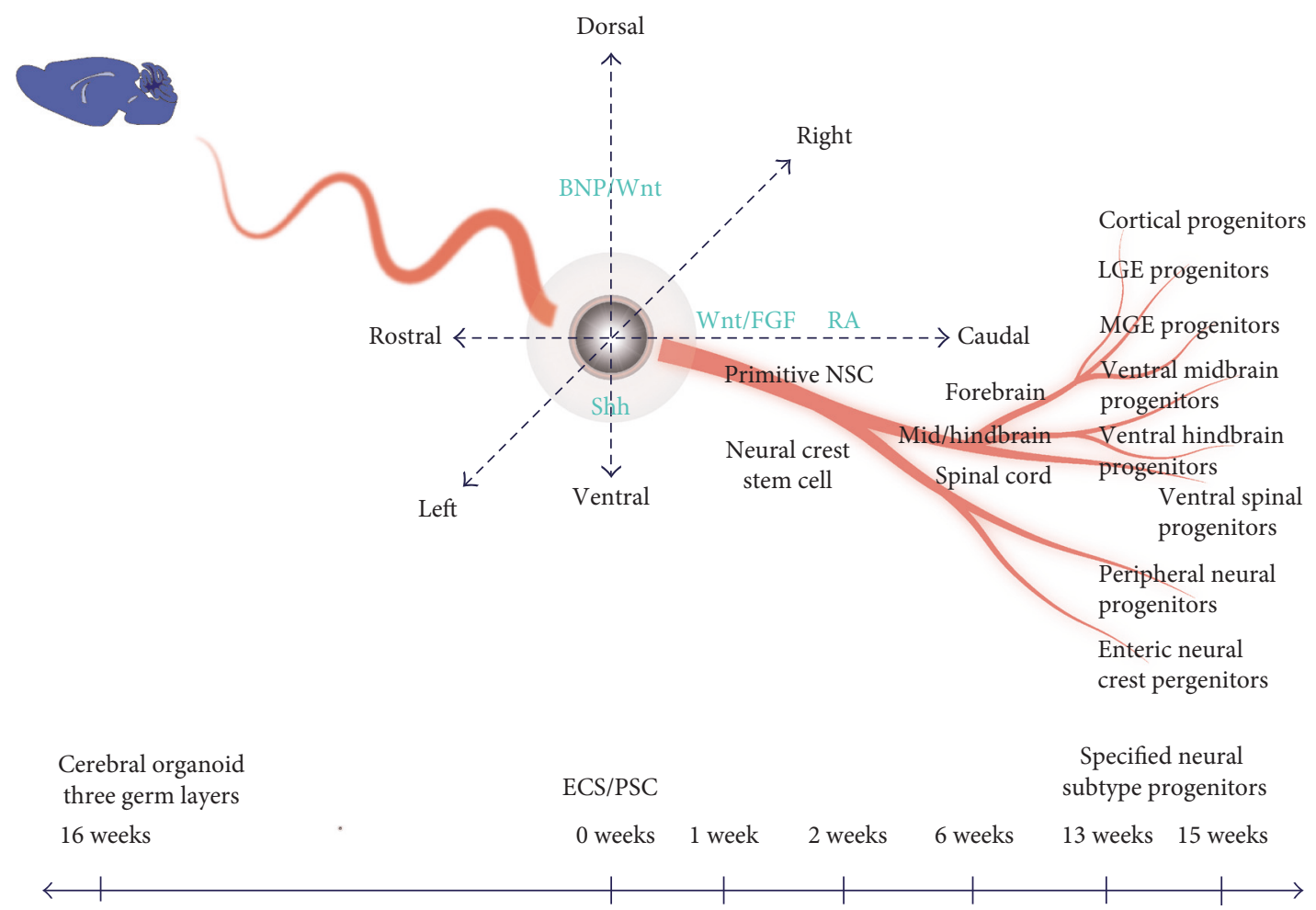

Figure 1: Steered with the specific spatiotemporal control strategies, a single embryonic stem cell (ESC) or pluripotent stem cell (PSC) can develop to a three germ layer brain and pure neural type. The outline has been depicted in Tao's paper (Tao et al.). BNP: bone morphogenetic protein; ESC: embryonic stem cell; FGF: fibroblast growth factor; LGE: lateral ganglionic eminence; MGE: medial ganglionic eminence; NSC: neural stem cell; PSC: pluripotent stem cell; RA: retinoic acid; Shh: sonic hedgehog.

therapy renders an obvious advantage over traditional stem cell therapy. Besides, this method focuses on fullfunctional organ-like tissue transplantation rather than purified neural cell type treatments. After engrafting into the host, they still have a stable environment in situ and can support themselves for self-renew and self-organize to integrate with host tissue. Thus, stem cells in the organoids have a higher survival rate and form functional connections with the surrounding tissue in the host [4, 7-9].

In recent decades, neural organoid has entered into and captured our eyes. Lancaster and his team successfully established a protocol for culturing pluripotent stem cell- (PSC-) derived "cerebral organoids" that recapitulated the developing human brain's cellular organization segregates into distinct brain regions [10]. Although cerebral organoids could not fully model the organization of the brain, the method still shed a light for future treatment of diseases through organoid system transplantation which can be established in vitro culturing. In addition to Lancaster's team, several other teams developed region-specific neural organoid such as the neocortex [11], telencephalon [12], cerebellar [13], neural tube [14], pituitary gland [15], hippocampus cortex [16], optic cup [17], neural retina [18], and inner ear sensory epithelial tissue. Single embryonic stem cells (ESCs) or PSCs can be self-organized to form three-layer cerebral organoid but can also be directed to develop a region-specific neural organoid.
Furthermore, they can also be manipulated and assembled to form specific morphogenesis organ (Figure 1). In specific spatiotemporal control conditions, scientists have directed ES or PSC to differentiate into both neuronal subtypes and glial subtypes. Neuronal progenitors can be specified into GABAergic, glutamatergic neurons, dopaminergic neurons, interneurons, and motoneurons [19-26], while glial progenitors can be specified into astrocytes, oligodentrocytes, and other glial subtypes [19, 27-29]. It is worthwhile mentioning that special signals can also be utilized to enhance the acquisition of the transmitter phenotype $[19,23]$. These findings stretch a promising panorama for clinical treatment by distinct organoid system transplantation. Over the past decades, scientists have devoted themselves to elucidating critical element brain development and spatiotemporal control of the processes, which are extensively and fully reviewed in several perfect papers $[5,19-21,27,30-35]$. These findings provide us with rationale and logistical feasibility to steer organogenesis to specific region. In addition, we can also design and assemble organoid to form specific morphology or function through manipulating numbers of specific stem cell types, neural network composition, numbers of receptors, and ligands. These organoids could be applied to treat central nervous system (CNS) diseases.

Although the classification of organoid was reviewed in previous papers [3], the authors focused on the purpose of 
organoid researches rather than the approaches to organoid formatting. Based on methods and application orientations, present review categorizes the organogenesis into three approaches. According to distinct procedure in the induction of organoid organization, we categorize these researches into three approaches (Table 1): (a) stepwise, direct organization of region-specific or population-enriched organoids $[12,18,36-47]$; (b) assemble and coculture distinct organspecific progenitor cells or stem cells to form specific morphogenesis organoid $[7,8,13,17,26,48]$; and (c) assemble embryoid bodies for induction of full layer organoid $[14,49,50]$. Besides, it will also provide with details of the examples and discuss on the rationale and logistical feasibility. In the following parts, we also compare distinct approaches and analyze for their possible indications for diseases.

\section{Neural Organogenesis Approach}

2.1. Stepwise, Direct Organization of Region-Specific or Population-Enriched Organoids. Currently, most researches on neural organogenesis adopt stepwise spatiotemporal control strategies to acquire neural organoids from ESCs or IPSCs [12, 18, 36-47]. In the initial stage, ESCs or IPSs are allowed to reaggregate in low-adhesion condition, namely, serum-free EB-like protocol (SFEBq), which ensures that they have enough time to proliferate and expand $[47,51]$. In this stage, ESCs or IPSCs maintain pluripotency and EBlike masses harbor three germ layers (ectoderm, mesoderm, and endoderm). In the stage of neural induction, referring to neuroectodermal formation, EB-like masses are transferred to N2 medium to induce neural germ layers. Treating with exogenous signal inhibitor of BMP, Wnt, and nodal inhibitor, they can efficiently form neuroepithelial tissue, neural tube construct [14], or neocortex [42]. The early neural organoids usually display initial structure and morphology with apical-basal polarity and dorsal-ventral polarity. Further induction can promote region identity and acquire region-specific organoids. Human cerebral cortex is well structured with six layer neurons. Deep and superficial layers of neurons are distinct populations, which are connected with each other and have distinct projections and functional fate $[33,52]$. As a result, with a good master of region-specific neural organoid induction technology, we can prepare specific population of progenitor cells which we want to perform cell replacement therapy. Although we cannot get purified cell population, high number of specific neural population can be acquired through using this method $[13,23,39,42]$. Thus, this organogenesis approach will be neural type population orientated.

With the support of specific spatiotemporal control strategies, this approach efficiently directs ESCs or PSCs differentiate into and self-organize region-specific neural organoids with a high number of specific progenitor cells (Figure 2(a)). Both intrinsic and extrinsic signals are involved in the regulations. During embryonic days 9 and 10 , corticogenesis in mice takes places in a polarized epithelium with its apical surface forming the lumen of the tube (future ventricles). Early cortical neural stem cells
(NSCs) divide symmetrically. At E11, NSCs begin to divide asymmetrically. One daughter cell retains its NSC identity while the other becomes a neuron. Early-born neurons form the deep layers of the cortical plate (layers 5 and 6), and later-born neurons migrate outward past the deep layers to establish the superficial or upper layers (layers 2-4). Although this neural induction process seems to be a cell fate program, it could be manipulated by this approach. For example, Muguruma et al. [13] reported that they acquired polarized cerebellar plate in 3D culture with a stepwise spatiotemporal control strategy. Firstly, they dissociated ESCs at day 0. In order to promote neuroectodermal differentiation, they inhibit mesenchymal differentiation by addition of the transforming growth factor $\beta$ - (TGF- $\beta$-) receptor blocker. On days $2-14$, ESCs were treated with FGF2 and insulin with the aim to be steered to differentiate into cerebellar progenitors. On day 14, additional FGF19 and SDF1 treatments induced progenitors to self-form cerebellar plate neuroepithelial structures with dorsal-ventral polarity. After these treatments, neuroepithelial rosettes had transformed into large and continuous flat-oval structures with the apical side inward regarding the ova. Admittedly, major portion of the cerebellar plate neuroepithelium generates Purkinje cells and interneurons and they finally acquired those electrophysiologically functional Purkinje cells.

Muguruma et al.'s success displays a good example which shows how scientists manipulate lineage of organogenesis. However, it will be the tip of an iceberg in the future. Recently, considerable excellent review papers have mapped neural subtype specification lineage and fundamental developmental principles [19-21, 28, 30, 32, 53]; based on which, we can briefly conclude as following: (1) Early cortical neural stem cells (NSCs) residing in a polarized epithelium divide symmetrically at their early expansion. At E11 in mice, NSCs begin to divide asymmetrically, generating one neuronal progenitors and the other continuing maintaining NSC identity. In this stage, apical surface forming the lumen of the tube (future ventricles), early neural progenitors migrate up and down within the ventricular zone (VZ) of the neuroepithelium. Neuronal daughters detach and migrate to subventricular zone (SVZ). (2) Although neocortical excitatory or inhibitory neurons can be generated in both VZ and SVZ, different regulator factors still determine their subtypes. Cux 2 and Cux2+ excitatory progenitors, respectively, generate distinct subtypes of upperlayer and deep-layer neurons. SST + or $\mathrm{PV}+$ progenitors result in inhibitory neurons in all layers except layer I whereas $\mathrm{CR}+$ or VIP+ cells give rise to inhibitory neurons particularly abundant in layers IV, III, and II. NPY+-derived cells could be found in all cortical layers; transcription factor FOXA2 is critical for midbrain DA neuron development while coexpressions of the floor plate (FP) marker FOXA2 and the roof plate marker LMX1A are as well required. (3) Astrocytes in the cerebral cortex are produced from the cortical ventricular zone (VZ) or from the ventral forebrain. In addition, glia of the cerebral cortex is also produced from the postnatal SVZ, a specialized reservoir of glial and neuronal progenitors. Almost all of 


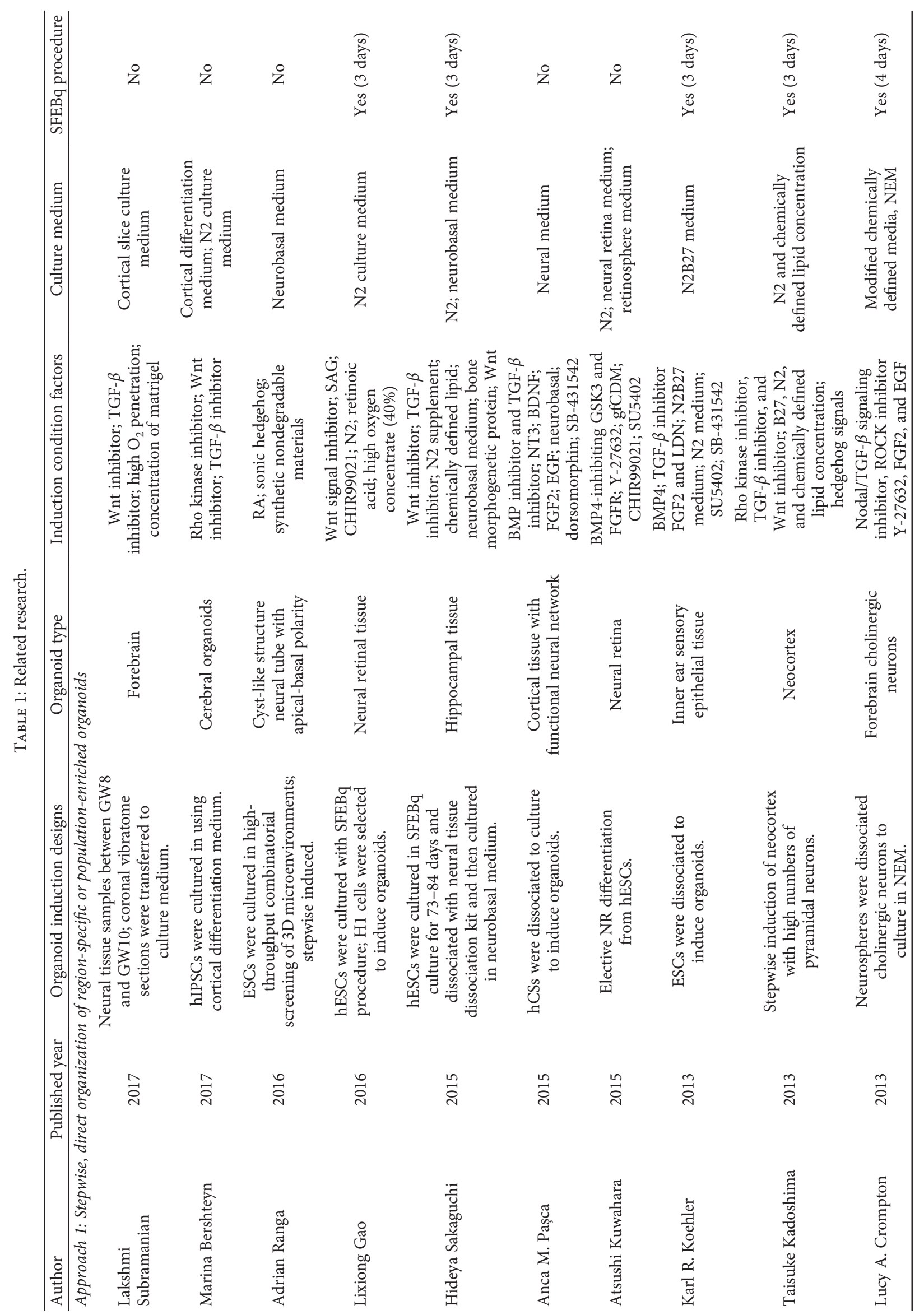




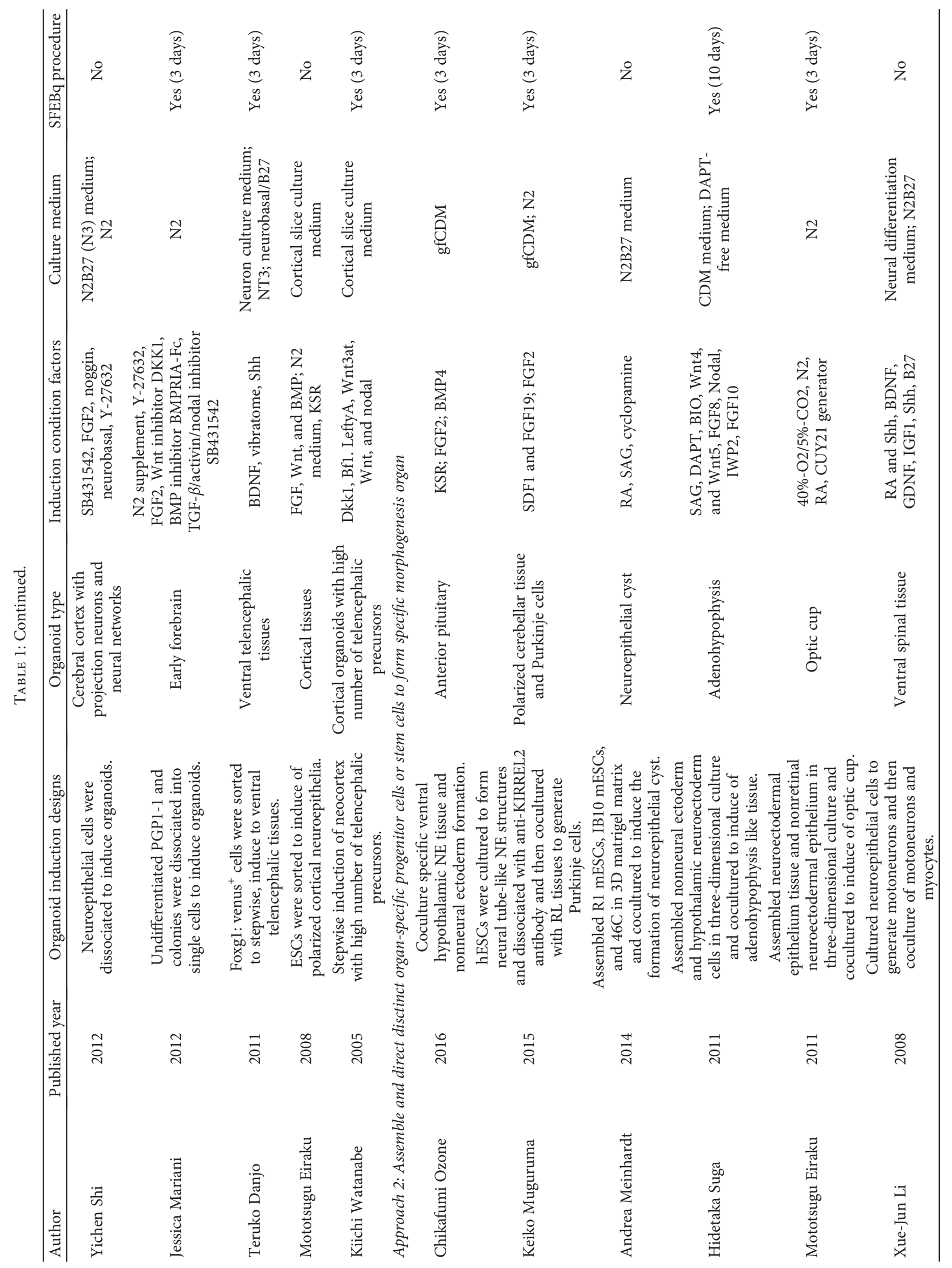




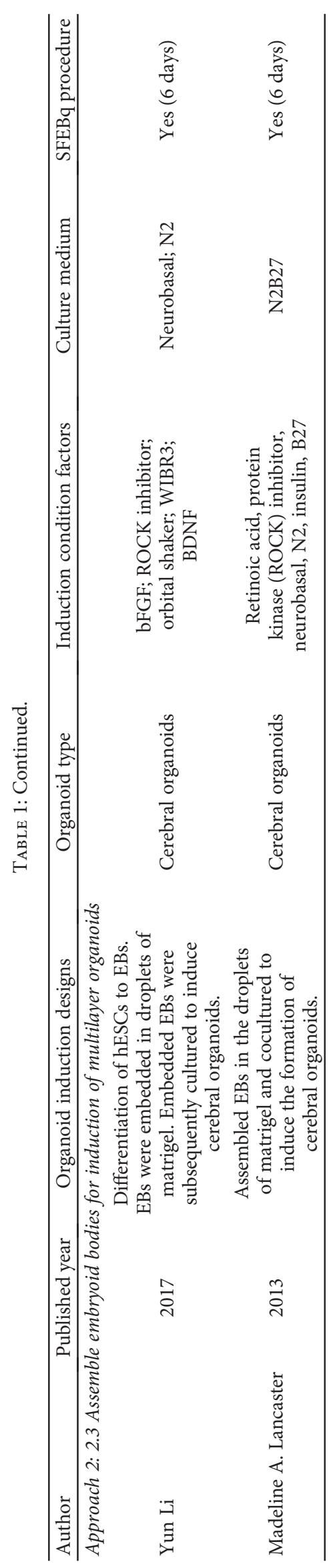




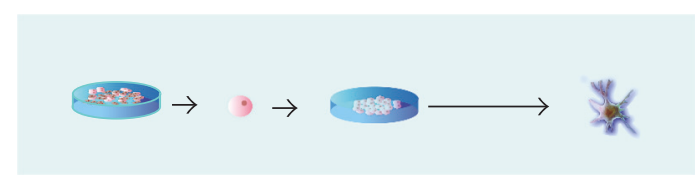

(a)

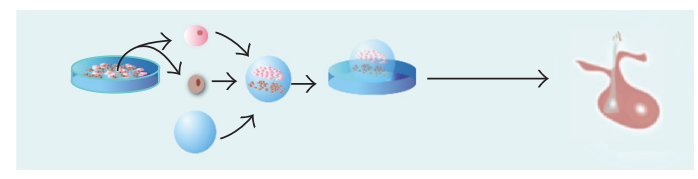

(b)

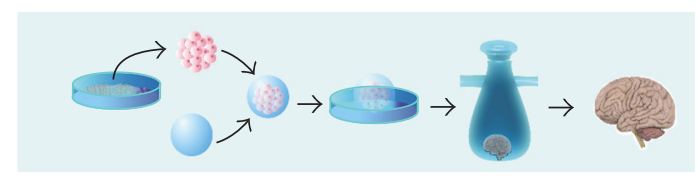

(c)

FIgURE 2: Schematic of three neural organogenesis approaches in vitro. (a) Stepwise, direct organization of region-specific or population-enriched organoids; (b) assemble and direct distinct organ-specific progenitor cells or stem cells to form specific morphogenesis organ; (c) assemble embryoid bodies for induction of multilayer organoids.

the neural subtype specification can be mapped in recent year researches [19] and could be manipulated to induce what we want (Figure 1).

Neural organogenesis via this approach is always neural population orientated. Also, it is expected to efficiently acquire specific neural progenitors or stem cells after region-specific differentiation induction. The regionspecific identity, regulated by cell surface signals, is important for neural network reconstruction [54] in the process of neuronal self-recognition and non-self-discrimination. Differentiation is directed by series of distinct epigenetic mechanisms [55-57]. In the stage of stepwise induction, ESCs or IPSs gradually lose their differentiation pluripotency due to DNA methylation and losing GC [56]. Distinct region specificity may have distinct cell identity. Although these specific neural stem cells are not purified, they still maintain their region identity ability [55]. The neurons with the same specific region identity easily connected with each other [58]. After they are engrafted to the specific region in vivo, they might easily establish neural network with local neurons. By contrast, neurons without an additional common factor have to take time to reconstitute their neural network. Compared with purified stem cell population transplantation, this population-enriched organoids may have a high-survival rate and efficiency to form mature synapse connections.

However, the organoids lost the potentiality to form multiple germ layer structure because stepwise differentiation induction steers the series of transcriptional regulators and DNA methylation to specific germ layer structure. Although stepwise strategy can induce initial neural cystic formation, this method might not construct sophisticated morphology efficiently [48]. Majority of these organoids can only form simple structures.
2.2. Assemble and Direct Distinct Organ-Specific Progenitor Cells or Stem Cells to Form Specific Morphogenesis Organ. This approach has been extensively applied to generate organoids with complex morphology, such as the pituitary $[7,8]$, optic cup [17], feather buds [59], salivary gland [60], hair follicle [61, 62], gingival tissues [63], and tooth [64]. These tissues usually locate in the transition region among distinct structure layers, and their organoid formation requires communication among the distinct region tissues. However, one induction condition only steers specific region identity. In order to acquire these organoids with complex architecture, they assemble two or more distinct populations of progenitors or stem cells in the 3D matrix and coculture under a specific differentiation induction condition $[7,8,13,17,26,48]$. This approach is defined as a term of "self-assembly" by Sasai [65]. Briefly, it refers to the spontaneous formation of a patterned organ with multistructure and multicellular by selective aggregation of cells or by rearrangement of the relative positions of cells within the structure [65]. Through assembling two or more cell types in a 3D culture, this method is sought to recapitulate an interactive microenvironment and mimic multicellular or multistructure level in the vivo organogenesis. For example, pituitary gland consists of neurohypophysis and adenohypophysis (Figure 2(b)). Adenohypophysis (anterior and intermediate lobes of the pituitary gland) contains several types of endocrine cells while neurohypophysis (posterior pituitary) consists of the axons and secretory termini of hypothalamic vasopressin and oxytocin neurons. In order to resemble structure of the pituitary gland, Suga et al. [8] detached outer component of epithelium cells of day 6 aggregates and cocultured them with inner neuroepithelial cells treating with hedgehog signaling. They found that this synthetic approach could successfully generate pituitary endocrine cells. At the interface of these two epithelia, Rathke's-pouch-like three-dimensional structures generated earlier. Functional organ bud was constructed in vitro, which was proved by the evidence that various endocrine cells efficiently secreted hormone in response to corticotrophin-releasing hormone after grafted in vivo. Based on the same approach, Eiraku's team achieved another success by reconstructing functional optic cup in vitro [17] and mimicking the multistructures of the optic cup consisting of the outer (pigmented) and inner (neurosensory) layers of the retina.

In addition to the pituitary gland and optic cup, the cortex in the central nervous system (CNS) also illustrates a prime example of an organ with extreme neuronal diversity and multilayer structures. Cell types of the cortex are broadly classified into excitatory projection neurons (PNs) and inhibitory interneurons (INs). This approaches might be applied to assemble PNs and INs in ratio, mimicking the vivo structures so as to allow enhanced cortical plasticity in the corticogenesis. Moreover, the process of spontaneous formation of ordered patterns and structures from a population of elements promotes functional connection with each other. Through bridging connections among neurons, glial cells and the vasculature, astrocytes provide with microenvironment and homeostatic processes for neuronal regeneration. Coculture astrocyte progenitors and neural stem cell 
might promote neurogenesis and synaptic connections [66-68]. Pouchelon et al. [69] found that functional differentiation of postsynaptic L4 neurons and cognate intracortical circuits were associated with TC-input-type-specific control. In addition, the finding also instructs the development of modality-specific neuronal and circuit properties during corticogenesis and shows another example of interactive communications among cellular levels. Due to these evidences, assembling multiprogenitors or differentiated PSCs or ESCs facilitates neurogenesis and functional connections. The approach appears more suitable for the organogenesis, requiring multicellular or multistructure interactive communications.

Assembling neural subtypes in the neural organoids also plays an important role because also it is important to reprogram the subtype diversity so as to promote the generation of functional neural circuit in the selforganization tissues. Distinct projecting neurons choose highly selective synaptic connectivity, both pre- and postsynaptic, within the same local circuits [70]. Both postsynaptic target of inhibitory interneurons and the properties of their synaptic connections depend on the identity of their projection partners [70]. Emerging data demonstrate that projection neurons and interneurons might "chemical match" for the development of excitatory and inhibitory cell assemblies [20]. Meanwhile, synaptic input also has the capability to affect specific neuron subtype differentiation during cortical circuit assembly [69]. Astrocytes comprise up to $40 \%$ of all CNS cells, which not only provide support to neurons but also actively regulate synapse formation and maturation [71]. Consequently, it appears a critical role of the assembling way of specific neural subtypes in establishing the neural circuit in the organoid.

\subsection{Assemble Embryoid Bodies for Induction of Multilayer} Organoids. Figure 2(c) illustrates procedures of this approach. After 4-day suspension culture, ESCs or PSCs aggregate and form embryoid/embryoid-like bodies. The procedure in vitro culture recapitulates the key events of embryogenesis in vivo to obtain the three developmental germ layers from which all cell types arise [4, 17, 50, 72]. The cell pellets are entrapped in a droplet of matrigel or collagen to coculture for and differentiate to develop a specific organ in a specifying differentiation strategy. Through manipulating extrinsic signal modulation, scientists can germ layer specification and cell differentiation [73]. In addition, embryoid bodies with three germ layers could also differentiate into functional tissue-specific cells with three germ layers. Takashi Tsuji's team designed a clustering-dependent embryoid body transplantation plan to develop a $3 \mathrm{D}$ integumentary organ system. In the system, formation involves three germ layers of cell types, respectively, dermis, hair follicles and sebaceous glands. After transplantation, hair follicles successfully generated with fine connections with the surrounding tissues such as the epidermis, arrector pili muscles, and nerve fibers, without tumorigenesis. Takagi et al.'s work provides not only a good example for assembling embryoid body approach but also an example for future application orientation that it appears to be suitable for the organogenesis involving more than one germ layer.

Different from other organoid induction approaches, this approach resorts to acquire specific organoid with full layer structure. Researchers adopt this approach to investigate natural organ development procedures or mechanisms involving diseases $[4,17,50,72]$. Unlike EB-like aggregation in SFEBq procedure, this approach prolongs the culturing time of EB-like population. Additionally, they assemble and coculture EB-like populations in $3 \mathrm{D}$ matrix in order to induce self-organization and morphogenesis.

At present, only a few neurological scientists focus on neural organogenesis or cerebral organoid with multiple germ layers [50] because the overwhelming majority of them hold the theory that neural organ induction starts in neuroectodermal stage. Lancaster et al. developed a cerebral organoid in vitro based on this method. Cerebral organoids showed recapitulate features of human cortical development, namely, characteristic progenitor zone organization with abundant outer radial glial stem cells. Most brain tissues derived from neuroectodermal layer whereas mesoderm and endoderm germ layers involve neural organogenesis. Formation of three germ layers cannot be isolated from each other. The germ layers are defined by their position at the stage of late gastrula. At the late stage of embryogenesis, their regional divisions are no longer distinct [74]. Cardiovascular and cerebrovascular derived from mesoderm germ layer stretch throughout the body including the brain and transport blood and energy. Nervous system originated from neuroectoderm forms parasympathetic and sympathetic nervous systems and governs the function of the cardiovascular system [75]. Therefore, they are supported by each other and connected with each other. Mesenchymal stem cells derived from mesoderm germ layer could also be applied in the degenerative neurological diseases [76-78]. It was found that human mesenchymal stem cells (hMSCs) in culture could provide humoral signals that selectively promote the genesis of neurons and oligodendrocytes from NSCs [68]. In addition, MSC could differentiate into neuron-like cells as well as by a competence to generate a "neuroprotective" environment [79]. This approach may facilitate local reconstitution of vascular networks. Considering the above discussion, we can make a speculation that the assembling embryoid body approach might be applied to generate a cerebral organoid with multiple germ layers. The organoid is more probably suitable for treating patients with multilayer brain tissue loss including traumatic brain injury, stroke [80], hemispherectomy, or lobotomy because of tumor, epilepsy, and intracranial hematoma.

\section{Similarities and Differences}

3.1. Similarities. Neural organogenesis is regulated by a series of epigenetic regulators. In order to develop to an organoid, a single cell in all approaches has to undergo spatiotemporal steering process. Neurons differentiate and migrate to specific regions and layers along anteriorposterior (AP) and dorsal-ventral (DV) axis [5] and are 
regulated by various regulator factors. Wnt, FGF, and retinoic acid (RA) are responsible for their caudalizing activity in the embryological context; Shh signaling for ventralization of embryonic neural tissue; BMP and Wnt signaling for dorsalization [5]. Besides, time order also determines the locations of neurons. Pioneer neurons are the earliest-born neurons in the cortex and then followed by deep cortical layers VI and V, then by upper layers IV, and lastly layers II/III [81]. Late-born neurons tend to localize more basally to early-born neurons [11]. All the three approaches possess the common epigenetic regulating factors. Even though neural organoids in the three approaches are different from each other in composition and structure in the organoids, they have a common neuroectodermal induction process.

In addition, all approaches adopt 3D culture to mimic in vivo microenvironment to provide a scaffold and niche for stem cells to aggregate, attach, and form organoids. Biochemical and biophysical signals are also involved to steer organogenesis in all three approaches. These signals determine organogenesis microenvironments consisting of a complex array of signaling mechanisms from niche support cells, the ECM, and mechanical forces, as well as systemic and physiochemical conditions such as oxygen and $\mathrm{pH}$ levels [82]. For example, the identity of PSCs is associated with local oxygen concentration and hypoxia inducible factor- $1 \alpha$ (HIF- $\alpha$ ) plays a distinct and stage-specific roles in reprogramming human cells to PSCs [83] and involves in angiogenesis and stem cell maintenance. NSCs within the SVZ maintain the integrity of their vascular niche through HIF-1-mediated signaling mechanisms [84]. Relief of hypoxia in developing the cerebral cortex by growth of blood vessels temporospatially coincided with NSC differentiation [85]. Considerable biophysical factors such as adhesion and viscoelastic and stress relaxation of extracellular matrices take impact not only on cell spreading and proliferation but also on the differentiation to specific cell types [86-89]. Biophysical cues also generate a change in protein conformation in response to tension or compression and thus to take effect on the cell formation [87]. All of these signals could be manipulated for lineage of specific organ. Currently, a three-dimensional culture is widely applied in organogenesis. In 3D organoid culture system, it allows the formation of brain tissues through either self-assembly or active induction. Some scientists attempt to display several subtype stem cells in ratio or in multilayer in order to mimic the ratio or structures in vivo and finally acquired full functional organ [4, 61]. With the support of $3 \mathrm{D}$ organoid culture, scientists have the possibility and opportunity to rewrite the structure or composition of organogenesis program in vitro.

3.2. Differences. Neural organoids via the first approach are specific region orientated. ESCs or IPSCs can be stepwise, induced to differentiate into an organoid with high number of neural populations. These populations of neural stem cells are not purified cells. Instead, the organoids consist of several region-specific neural populations with special cell surface marker. These cells can form specific morphology and structure $[18,39,40]$. Additionally, they can organize local neural connections among distinct populations [13, 40]. By treating with specific markers, neural organoids could be dissociated to collect purified stem cells or progenitor cells. Therefore, we can efficiently acquire purified neural cell populations with region identity via the first approach induction.

Via the second approach, neural organoids are specific morphology orientated. The organoids usually consist of several anatomic parts. Anatomically, the morphogenetic self-organization locates in the cross-connection area among distinct regions and requires coculture of distinct populations of neural cell populations. Coculture can steer these parts to generate functional and morphologic connections. In order to promote the morphogenesis, distinct populations with different region identity were assembled in 3D droplets. As a result, the ratios among distinct populations, the matrix composition, biophysical, and biomechanical parameters need to be designed precisely mimicking in vivo process. This approach has made higher requirements for assembling protocol. However, this neural organoids have specific indications for diseases. The organoids could be engrafted into the brain as an integral preorgan. However, size and morphology of these artificial organoids have to match with host tissue. Otherwise, they are being potential occupying lesion.

Neural organoids via the third approach are full-germ layer orientated. They have more complex and full structure and morphology. At present, researches focus on the development of brain structure $[14,49,50]$ and few of them have successfully mimicked the brain structure generation in vivo although $\mathrm{Li}$ et al. reported a folded cerebral organoid with simple structure [49]. This approach aims to acquire not only full function but also both integral structure and morphology. Cell populations in the neural organoids involve not only neural populations but also cell populations derived from other germ layers such as vessels and the immune system. However, how to reconstruct the cerebrovascular and immune system in the organoids still remains to be solved.

\section{Clinical Treatment Consideration}

4.1. Region-Specific or Population-Enriched Organoids. Stem cell therapy is a promising approach to replace damaged cells in the brain or replenish losing cells in the nucleus [90-92]. In a variety of neuronal degenerative diseases, patients have specific neural population loss or damaged. In Parkinson's disease (PD), midbrain dopamine (DA) neurons, especially innervating motor neurons, are degenerated at least at an early stage. However, Huntington's patients gradually lose their medium spiny GABA ( $\gamma$-aminobutyric acid) neurons in the striatum. Motor neuron loss could also be observed in spinal muscular atrophy (SMA) and amyotrophic lateral sclerosis (ALS) patients [19]. The specific neural subtypes are preferentially affected and degenerated, so few pharmacy drugs could curb the pathological insult progress. Neural stem cell therapy improves these diseases not only in animal models but also in clinical trials [77, 93-98]. Traditional purified cell therapy has low clinical efficiency. However, these neural populations generated by the first neural organoid induction approach could improve its treatment 
efficiency. These populations in the neural organoids have specific region identity. After engrafting in to the host brain, they could connect with local neurons in an efficient way. Thus, neural organoids via the first approaches are more suitable for these diseases with specific neural type loss or damage.

4.2. Assembled Specific Morphogenesis Organoids. These neural organoids are specific morphology orientated and theoretically suitable for treating these diseases with specific neural structure damaged or atrophy. For example, Sheehan's syndrome always follows after pituitary atrophy which results from postpartum bleeding and pituitary tumor or surgery $[99,100]$. Traditional treatment with pharmaceutical drug has several adverse effects. These patients might have another alternative treatment by transplantation of artificial pituitary induced by assembling and coculturing hypothalamic as well as oral ectoderm stem cells [7]. Other similar diseases can be optic atrophy [17], retinal diseases [18], and so on. In addition, peripheral nerves are other potential indications for the second neural organoids. Schwann cell in the peripheral nervous system is derived from the neural crest. Maturity of Schwann cell requires interaction among the Schwann cell and peripheral tissues [101]. Skin-derived precursor cells facilitate the regeneration process of peripheral nerve [102]. In addition, coculture of progenitor cells of peripheral tissues and neural stem cells might promote the generation of peripheral nerves.

4.3. Assembled Multilayer Organoids. Actually, these neural organoids are a preorgan with integral structure and function and can treat these diseases with structure loss or damage. These patients might have an integral structure loss of brain region because of traumatic brain injury, stroke, hemispherectomy, or lobotomy caused by tumor, epilepsy, and intracranial hematoma. There are no niches for stem cells to attach. Therefore, organoids have to support by themselves. Before engrafted to the host brain, the neural organoids must generate a preorgan with full structure.

\section{Conclusion}

To conclude, 3D organoid system transplantation renders obvious advantage over traditional approaches which probably focus on pure populations of particular stem cell-derived cell types. Instead, 3D organoid system resembles natural self-formation process of specific organ through assembling cell subtypes, layers, cell subtype proportion, and manipulating biophysical signals. These strategies promote correct connections among multilayer and multicellular synapses and establishment of local neural circuits. In comparison with conventional therapy, 3D organoid system transplantation promotes stem cell survival and functional connection after grafting in vivo $[4,8,17,61,64]$. Although 3D organoid system transplantation was reported to treat CNS diseases only in a few papers $[4,8,17,61,63,64]$, it still appears to be promising in the future treatment. There are three approaches in neural organoid which could be applied, choices of which can be determined depending upon due diseases. The first organogenesis approach is the region-specific or population-enriched organoids which refer to the fundamental method. We could acquire specific neural subtypes or specific organ, which could be applied to treat neuronal degenerative diseases, such as Parkinson's disease, Huntington disease, ALS, and SMA. The second synthetic approach designed in the multicellular level or multiculture level can generate functional self-formation tissue to treat neural organassociating functional disorders such as pituitary gland atrophy and optic cup loss. Peripheral nerve damage could also be treated by this organoids. The organogenesis approach by assembling embryoid bodies for specific organ is theoretically more suitable for patients with total layer tissue loss, such as traumatic brain injury, stroke $[80,103]$, hemispherectomy, and lobotomy because of tumor, epilepsy, and intracranial hematoma. In order to promote local functional connections, scientists should design the neural subtype diversity in the process of in vitro organoid induction, matching the ratio between excitatory and inhibitory neurons, neurons and astrocyte, and input synapses and output synapses.

\section{Conflicts of Interest}

The authors declare that they have no conflicts of interest.

\section{Authors' Contributions}

NaiLi Wei and ZiFang Quan contributed equally to this work.

\section{Acknowledgments}

This work was supported by the Grants (2013CB967400, 2012CB966300, 81641056, 81271003, and ZJ2014-ZD-002) from the National Nature Science Foundation and Ministry of Science and Technology of China and the Grant (15140902200) from the Shanghai Committee of Science and Technology.

\section{References}

[1] R. Y. Tam, T. Fuehrmann, N. Mitrousis, and M. S. Shoichet, "Regenerative therapies for central nervous system diseases: a biomaterials approach," Neuropsychopharmacology, vol. 39, no. 1, pp. 169-188, 2014.

[2] C. Zhao, W. Deng, and F. H. Gage, "Mechanisms and functional implications of adult neurogenesis," Cell, vol. 132, no. 4, pp. 645-660, 2008.

[3] M. A. Lancaster and J. A. Knoblich, "Organogenesis in a dish: modeling development and disease using organoid technologies," Science, vol. 345, no. 6194, article 1247125, 2014.

[4] R. Takagi, J. Ishimaru, A. Sugawara et al., "Bioengineering a 3D integumentary organ system from iPS cells using an in vivo transplantation model," Science Advances, vol. 2, no. 4, article e1500887, 2016.

[5] M. Eiraku and Y. Sasai, "Self-formation of layered neural structures in three-dimensional culture of ES cells," Current Opinion in Neurobiology, vol. 22, no. 5, pp. 768-777, 2012. 
[6] M. Bershteyn and A. R. Kriegstein, "Cerebral organoids in a dish: progress and prospects," Cell, vol. 155, no. 1, pp. 19-20, 2013.

[7] C. Ozone, H. Suga, M. Eiraku et al., "Functional anterior pituitary generated in self-organizing culture of human embryonic stem cells," Nature Communications, vol. 7, article 10351, 2016.

[8] H. Suga, T. Kadoshima, M. Minaguchi et al., "Self-formation of functional adenohypophysis in three-dimensional culture," Nature, vol. 480, no. 7375, pp. 57-62, 2011.

[9] Y. Sasai, "Next-generation regenerative medicine: organogenesis from stem cells in 3D culture," Cell Stem Cell, vol. 12, no. 5, pp. 520-530, 2013.

[10] S. M. Chambers, J. Tchieu, and L. Studer, "Build-a-Brain," Cell Stem Cell, vol. 13, no. 4, pp. 377-378, 2013.

[11] C. T. Lee, J. Chen, A. A. Kindberg et al., "CYP3A5 Mediates Effects of Cocaine on Human Neocorticogenesis: Studies using an In Vitro 3D Self-Organized hPSC Model with a Single Cortex-Like Unit," Neuropsychopharmacology, vol. 42, no. 3, pp. 774-784, 2017.

[12] K. Watanabe, D. Kamiya, A. Nishiyama et al., "Directed differentiation of telencephalic precursors from embryonic stem cells," Nature Neuroscience, vol. 8, no. 3, pp. 288-296, 2005.

[13] K. Muguruma, A. Nishiyama, H. Kawakami, K. Hashimoto, and Y. Sasai, "Self-organization of polarized cerebellar tissue in 3D culture of human pluripotent stem cells," Cell Reports, vol. 10, no. 4, pp. 537-550, 2015.

[14] A. Ranga, M. Girgin, A. Meinhardt et al., "Neural tube morphogenesis in synthetic 3D microenvironments," Proceedings of the National Academy of Sciences of the United States of America, vol. 113, no. 44, pp. E6831-E6839, 2016.

[15] S. W. Davis, A. H. Mortensen, J. L. Keisler et al., “ $\beta$-catenin is required in the neural crest and mesencephalon for pituitary gland organogenesis," BMC Developmental Biology, vol. 16, no. 1, p. 16, 2016.

[16] H. Sakaguchi, T. Kadoshima, M. Soen et al., "Generation of functional hippocampal neurons from self-organizing human embryonic stem cell-derived dorsomedial telencephalic tissue," Nature Communications, vol. 6, p. 8896, 2015.

[17] M. Eiraku, N. Takata, H. Ishibashi et al., "Self-organizing optic-cup morphogenesis in three-dimensional culture," Nature, vol. 472, no. 7341, pp. 51-56, 2011.

[18] A. Kuwahara, C. Ozone, T. Nakano, K. Saito, M. Eiraku, and Y. Sasai, "Generation of a ciliary margin-like stem cell niche from self-organizing human retinal tissue," Nature Communications, vol. 6, p. 6286, 2015.

[19] Y. Tao and S. C. Zhang, "Neural subtype specification from human pluripotent stem cells," Cell Stem Cell, vol. 19, no. 5, pp. 573-586, 2016.

[20] O. Marín and U. Müller, "Lineage origins of GABAergic versus glutamatergic neurons in the neocortex," Current Opinion in Neurobiology, vol. 26, pp. 132-141, 2014.

[21] G. Bartolini, G. Ciceri, and O. Marín, "Integration of GABAergic interneurons into cortical cell assemblies: lessons from embryos and adults," Neuron, vol. 79, no. 5, pp. 849864, 2013.

[22] A. L. Perrier, V. Tabar, T. Barberi et al., "Derivation of midbrain dopamine neurons from human embryonic stem cells," Proceedings of the National Academy of Sciences of the United States of America, vol. 101, no. 34, pp. 1254312548, 2004.
[23] X. J. Li, Z. W. Du, E. D. Zarnowska et al., "Specification of motoneurons from human embryonic stem cells," Nature Biotechnology, vol. 23, no. 2, pp. 215-221, 2005.

[24] P. Soundararajan, G. B. Miles, L. L. Rubin, R. M. Brownstone, and V. F. Rafuse, "Motoneurons derived from embryonic stem cells express transcription factors and develop phenotypes characteristic of medial motor column neurons," The Journal of Neuroscience, vol. 26, no. 12, pp. 3256-3268, 2006.

[25] J. T. Dimos, K. T. Rodolfa, K. K. Niakan et al., "Induced pluripotent stem cells generated from patients with ALS can be differentiated into motor neurons," Science, vol. 321, no. 5893, pp. 1218-1221, 2008.

[26] X. J. Li, B. Y. Hu, S. A. Jones et al., "Directed differentiation of ventral spinal progenitors and motor neurons from human embryonic stem cells by small molecules," Stem Cells, vol. 26, no. 4, pp. 886-893, 2008.

[27] G. Tyzack, A. Lakatos, and R. Patani, "Human stem cell-derived astrocytes: specification and relevance for neurological disorders," Current Stem Cell Reports, vol. 2, pp. 236-247, 2016.

[28] H. Tabata, "Diverse subtypes of astrocytes and their development during corticogenesis," Frontiers in Neuroscience, vol. 9, p. 114, 2015.

[29] M. R. Costa, O. Bucholz, T. Schroeder, and M. Götz, "Late Origin of Glia-Restricted Progenitors in the Developing Mouse," Cerebral Cortex, vol. 19, Supplement 1, pp. i135i143, 2009.

[30] S. Lodato, A. S. Shetty, and P. Arlotta, "Cerebral cortex assembly: generating and reprogramming projection neuron diversity," Trends in Neurosciences, vol. 38, no. 2, pp. 117-125, 2015.

[31] B. L. Finlay and R. Uchiyama, "Developmental mechanisms channeling cortical evolution," Trends in Neurosciences, vol. 38, no. 2, pp. 69-76, 2015.

[32] J. van den Ameele, L. Tiberi, P. Vanderhaeghen, and I. Espuny-Camacho, "Thinking out of the dish: what to learn about cortical development using pluripotent stem cells," Trends in Neurosciences, vol. 37, no. 6, pp. 334-342, 2014.

[33] S. Anderson and P. Vanderhaeghen, "Cortical neurogenesis from pluripotent stem cells: complexity emerging from simplicity," Current Opinion in Neurobiology, vol. 27, pp. 151157, 2014.

[34] K. Toma and C. Hanashima, "Switching modes in corticogenesis: mechanisms of neuronal subtype transitions and integration in the cerebral cortex," Frontiers in Neuroscience, vol. 9, p. 274, 2015.

[35] M. Peljto and H. Wichterle, "Programming embryonic stem cells to neuronal subtypes," Current Opinion in Neurobiology, vol. 21, no. 1, pp. 43-51, 2011.

[36] L. Subramanian, M. Bershteyn, M. F. Paredes, and A. R. Kriegstein, "Dynamic behaviour of human neuroepithelial cells in the developing forebrain," Nature Communications, vol. 8, article 14167, 2017.

[37] M. Bershteyn, T. J. Nowakowski, A. A. Pollen et al., "Human iPSC-derived cerebral organoids model cellular features of lissencephaly and reveal prolonged mitosis of outer radial glia," Cell Stem Cell, vol. 20, no. 4, pp. 435-449, 2017.

[38] L. Gao, X. Chen, Y. Zeng et al., "Intermittent high oxygen influences the formation of neural retinal tissue from human embryonic stem cells," Scientific Reports, vol. 6, article 29944, 2016. 
[39] H. Sakaguchi, T. Kadoshima, M. Soen et al., "Generation of functional hippocampal neurons from self-organizing human embryonic stem cell-derived dorsomedial telencephalic tissue," Nature Communications, vol. 6, p. 8896, 2015.

[40] A. M. Paşca, S. A. Sloan, L. E. Clarke et al., "Functional cortical neurons and astrocytes from human pluripotent stem cells in 3D culture," Nature Methods, vol. 12, no. 7, pp. 671-678, 2015.

[41] K. R. Koehler, A. M. Mikosz, A. I. Molosh, D. Patel, and E. Hashino, "Generation of inner ear sensory epithelia from pluripotent stem cells in 3D culture," Nature, vol. 500, no. 7461, pp. 217-221, 2013.

[42] T. Kadoshima, H. Sakaguchi, T. Nakano et al., "Selforganization of axial polarity, inside-out layer pattern, and species-specific progenitor dynamics in human ES cellderived neocortex," Proceedings of the National Academy of Sciences of the United States of America, vol. 110, no. 50, pp. 20284-20289, 2013.

[43] Y. Shi, P. Kirwan, J. Smith, H. P. Robinson, and F. J. Livesey, "Human cerebral cortex development from pluripotent stem cells to functional excitatory synapses," Nature Neuroscience, vol. 15 , no. 3, pp. 477-486, 2012, S1.

[44] L. A. Crompton, M. L. Byrne, H. Taylor et al., "Stepwise, non-adherent differentiation of human pluripotent stem cells to generate basal forebrain cholinergic neurons via hedgehog signaling," Stem Cell Research, vol. 11, no. 3, pp. 1206-1221, 2013.

[45] J. Mariani, M. V. Simonini, D. Palejev et al., "Modeling human cortical development in vitro using induced pluripotent stem cells," Proceedings of the National Academy of Sciences of the United States of America, vol. 109, no. 31, pp. 12770-12775, 2012.

[46] T. Danjo, M. Eiraku, K. Muguruma et al., "Subregional specification of embryonic stem cell-derived ventral telencephalic tissues by timed and combinatory treatment with extrinsic signals," Journal of Neuroscience, vol. 31, no. 5, pp. 1919-1933, 2011.

[47] M. Eiraku, K. Watanabe, M. Matsuo-Takasaki et al., "Selforganized formation of polarized cortical tissues from ESCs and its active manipulation by extrinsic signals," Cell Stem Cell, vol. 3, no. 5, pp. 519-532, 2008.

[48] A. Meinhardt, D. Eberle, A. Tazaki et al., “Tanaka EM.3D reconstitution of the patterned neural tube from embryonic stem cells," Stem Cell Reports, vol. 3, no. 6, pp. 987-999, 2014.

[49] Y. Li, J. Muffat, A. Omer et al., "Induction of expansion and folding in human cerebral organoids," Cell Stem Cell, vol. 20, no. 3, pp. 385.e3-396.e3, 2017.

[50] M. A. Lancaster, M. Renner, C. A. Martin et al., "Cerebral organoids model human brain development and microcephaly," Nature, vol. 501, no. 7467, pp. 373-379, 2013.

[51] C. J. Parr, S. Yamanaka, and H. Saito, "An update on stem cell biology and engineering for brain development," Molecular Psychiatry, vol. 22, no. 6, pp. 808-819, 2017.

[52] N. D. Dwyer, B. Chen, S. J. Chou, S. Hippenmeyer, L. Nguyen, and H. T. Ghashghaei, "Neural stem cells to cerebral cortex: emerging mechanisms regulating progenitor behavior and productivity," Journal of Neuroscience, vol. 36, no. 45, pp. 11394-11401, 2016.

[53] K. T. Sultan, W. Shi, and S. H. Shi, "Clonal origins of neocortical interneurons," Current Opinion in Neurobiology, vol. 26, pp. 125-131, 2014.
[54] K. M. Goodman, R. Rubinstein, C. A. Thu et al., "Structural basis of diverse homophilic recognition by clustered alphaand beta-protocadherins," Neuron, vol. 90, no. 4, pp. 709723, 2016.

[55] A. H. Rizvi, P. G. Camara, E. K. Kandror et al., "Singlecell topological RNA-seq analysis reveals insights into cellular differentiation and development," Nature Biotechnology, vol. 35, no. 6, pp. 551-560, 2017.

[56] W. Xie, M. D. Schultz, R. Lister et al., "Epigenomic analysis of multilineage differentiation of human embryonic stem cells," Cell, vol. 153, no. 5, pp. 1134-1148, 2013.

[57] L. Telley, S. Govindan, J. Prados et al., "Sequential transcriptional waves direct the differentiation of newborn neurons in the mouse neocortex," Science, vol. 351, no. 6280, pp. 1443-1446, 2016.

[58] G. Masserdotti, S. Gillotin, B. Sutor et al., "Transcriptional mechanisms of proneural factors and REST in regulating neuronal reprogramming of astrocytes," Cell Stem Cell, vol. 17, no. 1, pp. 74-88, 2015.

[59] K. Ishida and T. Mitsui, "Generation of bioengineered feather buds on a reconstructed chick skin from dissociated epithelial and mesenchymal cells," Development, Growth \& Differentiation, vol. 58, no. 3, pp. 303-314, 2016.

[60] M. Ogawa, M. Oshima, A. Imamura et al., "Functional salivary gland regeneration by transplantation of a bioengineered organ germ," Nature Communications, vol. 4, p. 2498, 2013.

[61] K. E. Toyoshima, K. Asakawa, N. Ishibashi et al., "Fully functional hair follicle regeneration through the rearrangement of stem cells and their niches," Nature Communications, vol. 3, p. 784, 2012.

[62] J. Fu and W. Hsu, "Epidermal Wnt controls hair follicle induction by orchestrating dynamic signaling crosstalk between the epidermis and dermis," The Journal of Investigative Dermatology, vol. 133, no. 4, pp. 890-898, 2013.

[63] H. Egusa, K. Okita, H. Kayashima et al., "Gingival fibroblasts as a promising source of induced pluripotent stem cells," PLoS One, vol. 5, no. 9, article e12743, 2010.

[64] K. Nakao, R. Morita, Y. Saji et al., "The development of a bioengineered organ germ method," Nature Methods, vol. 4, no. 3, pp. 227-230, 2007.

[65] Y. Sasai, "Cytosystems dynamics in self-organization of tissue architecture," Nature, vol. 493, no. 7432, pp. 318-326, 2013.

[66] Y. Yao, C. Huang, P. Gu, and T. Wen, "Combined MSCsecreted factors and neural stem cell transplantation promote functional recovery of PD rats," Cell Transplantation, vol. 25, no. 6, pp. 1101-1113, 2016.

[67] S. M. Hosseini, M. Farahmandnia, Z. Razi et al., "Combination cell therapy with mesenchymal stem cells and neural stem cells for brain stroke in rats," International Journal of Stem Cells, vol. 8, no. 1, pp. 99-105, 2015.

[68] L. Bai, A. Caplan, D. Lennon, and R. H. Miller, "Human mesenchymal stem cells signals regulate neural stem cell fate," Neurochemical Research, vol. 32, no. 2, pp. 353362, 2007.

[69] G. Pouchelon, F. Gambino, C. Bellone et al., "Modalityspecific thalamocortical inputs instruct the identity of postsynaptic L4 neurons," Nature, vol. 511, no. 7510, pp. 471-474, 2014.

[70] S. P. Brown and S. Hestrin, "Intracortical circuits of pyramidal neurons reflect their long-range axonal targets," Nature Biotechnology, vol. 457, no. 7233, pp. 1133-1136, 2009. 
[71] L. E. Clarke and B. A. Barres, "Emerging roles of astrocytes in neural circuit development," Nature Reviews Neuroscience, vol. 14, no. 5, pp. 311-321, 2013.

[72] G. Pettinato, X. Wen, and N. Zhang, "Engineering strategies for the formation of Embryoid bodies from human pluripotent stem cells," Stem Cells and Development, vol. 24, no. 14, pp. 1595-1609, 2015.

[73] G. G. Giobbe, F. Michielin, C. Luni et al., "Functional differentiation of human pluripotent stem cells on a chip," Nature Methods, vol. 12, no. 7, pp. 637-640, 2015.

[74] P. P. Tam and D. A. Loebel, "Gene function in mouse embryogenesis: get set for gastrulation," Nature Reviews Genetics, vol. 8, no. 5, pp. 368-381, 2007.

[75] V. Coskun and D. M. Lombardo, "Studying the pathophysiologic connection between cardiovascular and nervous systems using stem cells," Journal of Neuroscience Research, vol. 94, no. 12, pp. 1499-1510, 2016.

[76] C. Zhao, H. Li, X. J. Zhao et al., "Heat shock protein 60 affects behavioral improvement in a rat model of Parkinson's disease grafted with human umbilical cord mesenchymal stem cell-derived dopaminergic-like neurons," Neurochemical Research, vol. 41, no. 6, pp. 1238-1249, 2016.

[77] P. Petrou, Y. Gothelf, Z. Argov et al., "Safety and clinical effects of mesenchymal stem cells secreting neurotrophic factor transplantation in patients with amyotrophic lateral sclerosis," JAMA Neurology, vol. 73, no. 3, pp. 337-344, 2016.

[78] M. Villanova and J. R. Bach, “Allogeneic mesenchymal stem cell therapy outcomes for three patients with spinal muscular atrophy type 1," American Journal of Physical Medicine \& Rehabilitation, vol. 94, no. 5, pp. 410-415, 2015.

[79] C. Allers, J. A. Jones, G. P. Lasala, and J. J. Minguell, "Mesenchymal stem cell therapy for the treatment of amyotrophic lateral sclerosis: signals for hope," Regenerative Medicine, vol. 9, no. 5, pp. 637-647, 2014.

[80] Y. Yao, W. Miao, Z. Liu et al., "Dimethyl fumarate and monomethyl fumarate promote post-Ischemic recovery in mice," Translational Stroke Research, vol. 7, no. 6, pp. 535547, 2016.

[81] I. Espuny-Camacho, K. A. Michelsen, D. Gall et al., "Pyramidal neurons derived from human pluripotent stem cells integrate efficiently into mouse brain circuits in vivo," Neuron, vol. 77, no. 3, pp. 440-456, 2013.

[82] X. Yin, B. E. Mead, H. Safaee, R. Langer, J. M. Karp, and O. Levy, "Engineering stem cell organoids," Cell Stem Cell, vol. 18, no. 1, pp. 25-38, 2016.

[83] J. Mathieu, W. Zhou, Y. Xing et al., "Hypoxia-inducible factors have distinct and stage-specific roles during reprogramming of human cells to pluripotency," Cell Stem Cell, vol. 14, no. 5, pp. 592-605, 2014.

[84] X. Jiang, H. Pu, X. Hu et al., "A post-stroke therapeutic regimen with omega-3 polyunsaturated fatty acids that promotes white matter integrity and beneficial microglial responses after cerebral ischemia," Translational Stroke Research, vol. 7, no. 6, pp. 548-561, 2016.

[85] C. Lange, M. Turrero Garcia, I. Decimo et al., "Relief of hypoxia by angiogenesis promotes neural stem cell differentiation by targeting glycolysis," The EMBO Journal, vol. 35, no. 9, pp. 924-941, 2016.

[86] S. S. Ho, K. C. Murphy, B. Y. Binder, C. B. Vissers, and J. K. Leach, "Increased survival and function of mesenchymal stem cell spheroids entrapped in instructive alginate hydrogels," Stem Cells Translational Medicine, vol. 5, no. 6, pp. 773-781, 2016.

[87] S. W. Crowder, V. Leonardo, T. Whittaker, P. Papathanasiou, and M. M. Stevens, "Material cues as potent regulators of epigenetics and stem cell function," Cell Stem Cell, vol. 18, no. 1, pp. 39-52, 2016.

[88] O. Chaudhuri, L. Gu, D. Klumpers et al., "Hydrogels with tunable stress relaxation regulate stem cell fate and activity," Nature Materials, vol. 15, no. 3, pp. 326-334, 2016.

[89] O. Chaudhuri, L. Gu, M. Darnell et al., "Substrate stress relaxation regulates cell spreading," Nature Communications, vol. 6, p. 6364, 2015.

[90] L. Wei, Z. Z. Wei, M. Q. Jiang, O. Mohamad, and S. P. Yu, "Stem cell transplantation therapy for multifaceted therapeutic benefits after stroke," Progress in Neurobiology, no. 16, pp. 30115-30110, 2017.

[91] C. An, X. Jiang, H. Pu et al., "Severity-dependent long-term spatial learning-memory impairment in a mouse model of traumatic brain injury," Translational Stroke Research, vol. 7, no. 6, pp. 512-520, 2016.

[92] D. K. Smith, M. He, C. L. Zhang, and J. C. Zheng, "The therapeutic potential of cell identity reprogramming for the treatment of aging-related neurodegenerative disorders," Progress in Neurobiology, no. 15, pp. $30067-$ 30068, 2016.

[93] M. C. Moura, M. R. Novaes, Y. S. Zago, E. J. Eduardo, and L. A. Casulari, "Efficacy of stem cell therapy in amyotrophic lateral sclerosis: a systematic review and metaanalysis," Journal of Clinical Medicine Research, vol. 8, no. 4, pp. 317-324, 2016.

[94] H. Wei, Y. Li, S. Han et al., "cPKC $\gamma$-modulated autophagy in neurons alleviates ischemic injury in brain of mice with ischemic stroke through Akt-mTOR pathway," Translational Stroke Research, vol. 7, no. 6, pp. 497-511, 2016.

[95] I. Faravelli, M. Nizzardo, G. P. Comi, and S. Corti, "Spinal muscular atrophy-recent therapeutic advances for an old challenge," Nature Reviews Neurology, vol. 11, no. 6, pp. 351-359, 2015.

[96] H. Mitsumoto, B. R. Brooks, and V. Silani, "Clinical trials in amyotrophic lateral sclerosis: why so many negative trials and how can trials be improved?," The Lancet Neurology, vol. 13, no. 11, pp. 1127-1138, 2014.

[97] E. L. Feldman, N. M. Boulis, J. Hur et al., "Intraspinal neural stem cell transplantation in amyotrophic lateral sclerosis: phase 1 trial outcomes," Annals of Neurology, vol. 75, no. 3, pp. 363-373, 2014.

[98] B. Yang, K. Parsha, K. Schaar, X. Xi, J. Aronowski, and S. I. Savitz, "Various cell populations within the mononuclear fraction of bone marrow contribute to the beneficial effects of autologous bone marrow cell therapy in a rodent stroke model," Translational Stroke Research, vol. 7, no. 4, pp. 322-330, 2016.

[99] H. Diri, Z. Karaca, F. Tanriverdi, K. Unluhizarci, and F. Kelestimur, "Sheehan's syndrome: new insights into an old disease," Endocrine, vol. 51, no. 1, pp. 22-31, 2016.

[100] K. H. Darzy and S. M. Shalet, "Hypopituitarism as a consequence of brain tumours and radiotherapy," Pituitary, vol. 8, no. 3-4, pp. 203-211, 2005.

[101] A. Woodhoo and L. Sommer, "Development of the Schwann cell lineage: from the neural crest to the myelinated nerve," Glia, vol. 56, no. 14, pp. 1481-1490, 2008. 
[102] S. K. Walsh, T. Gordon, B. M. Addas, S. W. Kemp, and R. Midha, "Skin-derived precursor cells enhance peripheral nerve regeneration following chronic denervation," Experimental Neurology, vol. 223, no. 1, pp. 221-228, 2010.

[103] H. Pu, X. Jiang, X. Hu et al., "Delayed docosahexaenoic acid treatment combined with dietary supplementation of omega-3 fatty acids promotes long-term neurovascular restoration after ischemic stroke," Translational Stroke Research, vol. 7, no. 6, pp. 521-534, 2016. 

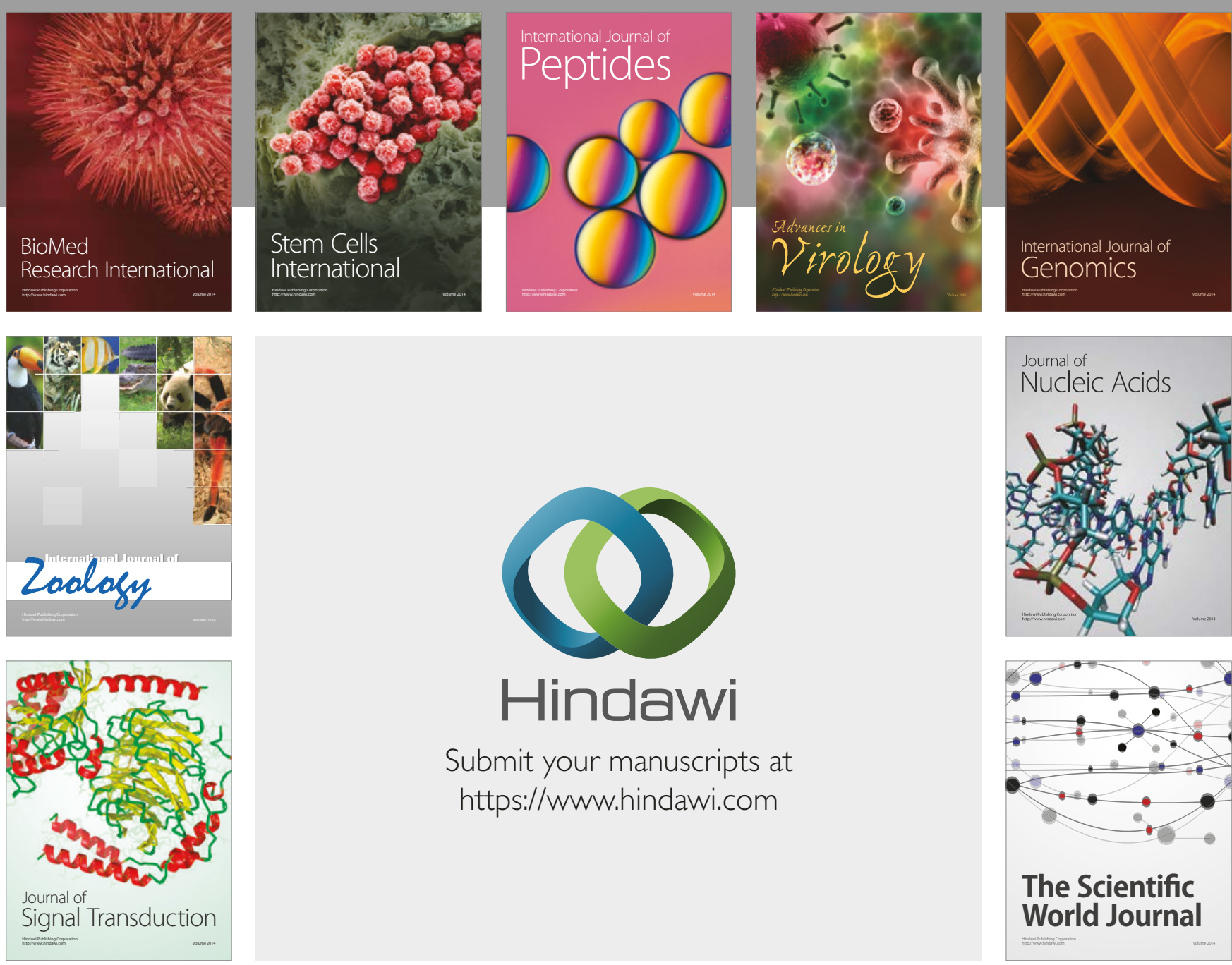

Submit your manuscripts at

https://www.hindawi.com
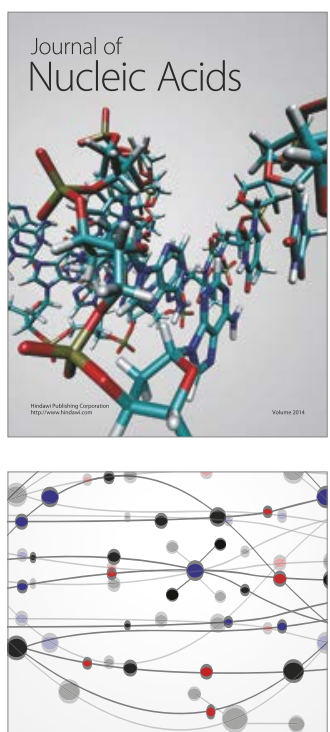

The Scientific World Journal

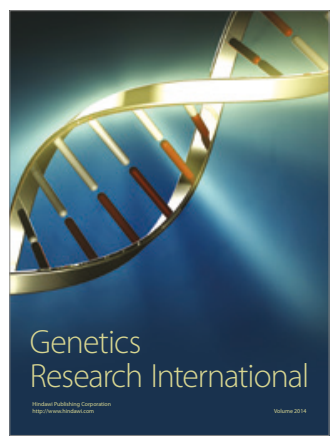

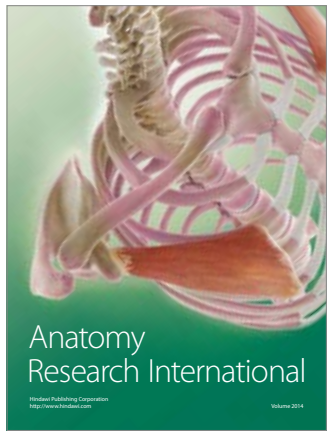

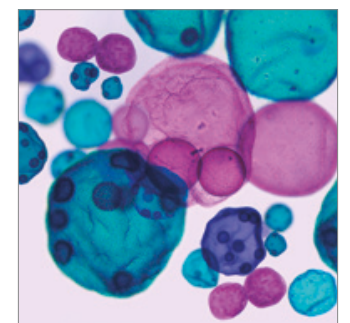

International Journal of Microbiology
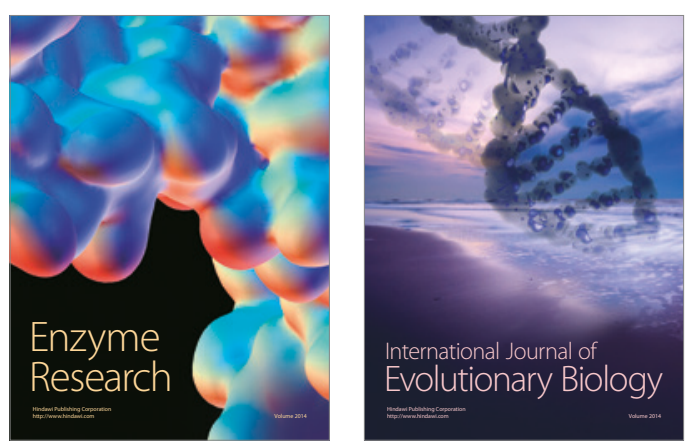
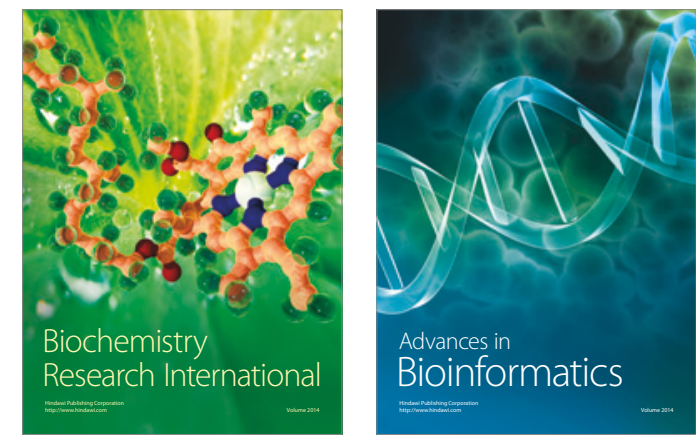

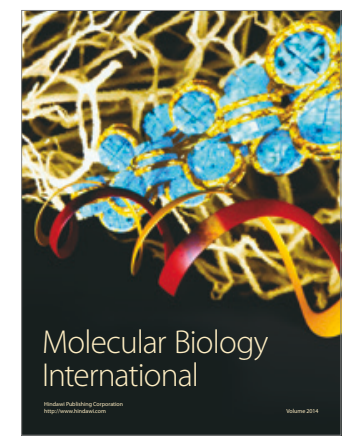

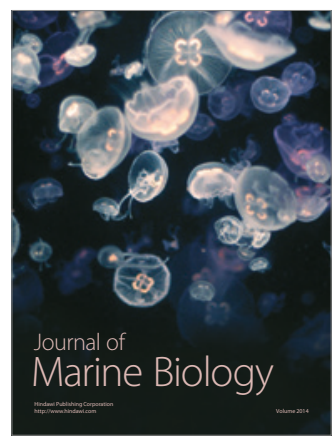

